\title{
Mass-Stiffness Combined Perturbation Method for Mode Shape Monitoring of Bridge Structures
}

\author{
Liye Zhang, ${ }^{1,2}$ Ye Xia $\mathbb{D}^{1},{ }^{1}$ Jose A. Lozano-Galant, ${ }^{3}$ and Limin Sun ${ }^{1,4}$ \\ ${ }^{1}$ Department of Bridge Engineering, Tongji University, Shanghai 200092, China \\ ${ }^{2}$ Research Institute of Highway Ministry of Transport, Beijing 100088, China \\ ${ }^{3}$ Department of Civil Engineering, University of Castilla-La Mancha, Ciudad Real 13071, Spain \\ ${ }^{4}$ State Key Laboratory for Disaster Reduction in Civil Engineering, Tongji University, Shanghai 200092, China \\ Correspondence should be addressed to Ye Xia; yxia@tongji.edu.cn
}

Received 26 April 2019; Revised 23 July 2019; Accepted 17 September 2019; Published 6 November 2019

Guest Editor: Franco Concli

Copyright (C 2019 Liye Zhang et al. This is an open access article distributed under the Creative Commons Attribution License, which permits unrestricted use, distribution, and reproduction in any medium, provided the original work is properly cited.

\begin{abstract}
Identification of the mode shapes through monitoring is one of the key problems in damage diagnosis based on modal parameters especially for damaged structures. In order to obtain mode shapes of damaged structures easily and accurately, the mass-stiffness combined perturbation (MSCP) method is proposed in this paper. To do so, the relationship between the stiffness perturbation mode shapes of damaged and intact structures is firstly derived and established. Then, the principle of similar frequency is applied to optimize the objective function of the most suitable mass perturbation model. Both numerical analyses and experimental tests on simple and complex structures demonstrate that the proposed MSCP method achieves higher precision than traditional mode shape identification methods. The additional advantages of the MSCP method include (i) lower requirement on the frequency analysis of only damaged structures and (ii) higher effectiveness for minor damage scenarios. In fact, the lower the damage, the higher the precision achieved by the MSCP method. As illustrated in the paper, the proposed technique has excellent applications in mode shapes identification and structural health monitoring.
\end{abstract}

\section{Introduction}

Damage assessment is an important subproblem of bridge health monitoring (BHM) that provides useful information for the decision-making process to maintain, repair, rehabilitate, or replace a structure [1-3]. Over the past three decades, changes in the modal parameters have been extensively used in damage assessment techniques [4-7]. In these methods, the vibration characteristics of the structures, such as natural frequency, mode shape, frequency response function, modal flexibility, mode shape curvature, and modal strain energy, are traditionally used to detect structural damages [8-10]. The vibration methods might be classified in terms of the data set used as frequency-based [11], mode shape-based [12], and the combination of both [13].

Frequency-based methods are based on the analysis of changes in natural frequencies [14-17]. The main advantage of this approach is its applicability as natural frequencies are relatively simple to monitor on site with high precision [18-22]. However, there are many limitations in the use of changes in the natural frequencies to structural damage identification [12, 23]. Firstly, natural frequencies mainly reflect the global structural response. For this reason, especially in larger structures, a significant damage may cause very small changes in natural frequencies. This reduced effect may be unnoticed during monitoring operations because of measurement noises and processing errors. Secondly, some environmental factors, such as temperature, may introduce uncertainties in the measured frequencies, leading to wrong damage identification results. Thirdly, natural frequencies might be affected by the material performance degradation, such as concrete carbonation, cracked concrete, or steel corrosion [24, 25]. Moreover, the efficiency of the frequency-based methods depends on the analytical finite element model (FEM) as well as the accuracy 
of the information measured on site [11]. Most studies in the literature assume that a nondamaged (intact) FEM is precise enough to simulate the vibration properties of the structures [26-30]. However, in reality, these models include a number of uncertainties, such as modeling and measurement errors, inaccurate physical parameters, idealized boundary conditions, and nonlinearity in the structural properties, which might play an important role in the simulation of the natural frequencies [31]. Therefore, it is widely believed that a change in natural frequencies alone might not provide enough information for efficient damage identification.

Alternatively to the frequency-based methods, the mode-shape approach assumes that omitting the changes in the mode shape adversely affects the accuracy of the damage detection algorithms [32, 33]. For this reason, effective damage indexes traditionally include mode shape information. Another advantage of this approach is the fact that mode shapes are more sensitive to identify local damages than natural frequencies. However, the use of mode shapes has also several drawbacks. Firstly, monitoring this information is more complex and relatively inaccurate, as the extracted mode shapes are commonly affected by environmental noises. Secondly, the number and location of the sensors might play an important role on the accuracy of the monitored mode shapes, and unfortunately, the size of the measurement set is usually significantly smaller than the one of the analytical model. Finally, the key problem of the vibration-based damage assessment methods is the monitoring and identification of mode shapes as relative changes of mode shapes are larger than those observed for natural frequencies [34]. To fill this gap, a convenient monitoring and identification method based on the mode shape analysis by the mass-stiffness combined perturbation (MSCP) method is proposed in this paper. Although the mode shapes are more sensitive to identify local damage, its monitoring is more complex and relatively inaccurate as it depends on the number and the location of the sensors. To solve this problem, a convenient monitoring and identification method is also proposed. To apply the MSCP method, the natural frequencies and mode shapes of the intact structure and mass perturbation structure should be obtained first through dynamic testing. The method only requires monitoring the frequencies of the damaged structure to perform damaged diagnosis with high accuracy and reduced monitoring cost. One of the main advantages of the MSCP method is its high effectiveness for minor damage scenarios. In fact, the lower the damage, the higher the precision achieved by the proposed procedure.

This paper is organized as follows: In Section 2, the proposed MSCP method is described. Firstly, the characteristic equations for the intact and damaged structural model are analyzed. Then, the relations between changes in mode shapes due to damages and mass perturbations are established. Next, the principle of similar frequency is applied to optimize the objective function for selecting the mass perturbation model. In Section 3, numerical and experimental analyses are used to demonstrate the effectiveness of the MSCP method. In Section 4, a continuous bridge is analyzed to illustrate the application of the proposed technique to real structures. Finally, the main conclusions are drawn in Section 5. The advantages of the proposed MSCP method encourage its implementation into health monitoring systems for damage identification in bridges.

\section{Mass-Stiffness Combined Perturbation Method}

2.1. Equations and Derivations. The characteristic equations of the intact and the damaged structure are as follows:

$$
\begin{aligned}
& \left(K^{u}-\lambda_{i}^{u} \cdot M^{u}\right) \cdot \phi_{i}^{u}=0, \\
& \left(K^{d}-\lambda_{i}^{d} \cdot M^{d}\right) \cdot \phi_{i}^{d}=0,
\end{aligned}
$$

where $K^{j}$ and $M^{j}$ are the global stiffness matrix and global mass matrix and $\lambda_{i}^{j}$ and $\phi_{i}^{j}$ are the $i^{\text {th }}$ eigenvalue (or frequency) and corresponding mode shape. These parameters define both the undamaged $(j=u)$ and the damaged $(j=d)$ structures, respectively.

Structural damage is considered the result of a change in the stiffness and the mass matrix. According to the perturbation theory $[35,36]$, the global stiffness and mass matrix for the damaged structure might be expressed as follows:

$$
\begin{gathered}
K^{d}=K^{u}+\Delta K, \\
M^{d}=M^{u}+\Delta M,
\end{gathered}
$$

where $\Delta K$ and $\Delta M$ denotes the changes in the global mass and stiffness matrix, respectively. As a change in stiffness or mass leads to changes in the modal characteristics, the eigenvalue $\Delta \lambda_{i}$ and its corresponding mode shape $\Delta \phi_{i}$ can be expressed as follows:

$$
\begin{aligned}
& \Delta \lambda_{i}=\lambda_{i}^{d}-\lambda_{i}^{u}, \\
& \Delta \phi_{i}=\phi_{i}^{d}-\phi_{i}^{u} .
\end{aligned}
$$

According to the perturbation method, the characteristic equation of the damaged structure presented in equation (2) might be expressed as follows:

$$
\left[K^{u}+\Delta K-\left(\lambda_{i}^{u}+\Delta \lambda_{i}\right)\left(M^{u}+\Delta M\right)\right]\left(\phi_{i}^{u}+\Delta \phi_{i}\right)=0 .
$$

Assuming a constant mass in the structure, equation (5) might be written as follows:

$$
\left[K^{u}-\lambda_{i}^{u} \cdot M^{u}+\Delta K-\Delta \lambda_{i, K} \cdot M^{u}\right]\left(\phi_{i}^{u}+\Delta \phi_{i, K}\right)=0,
$$

where $\Delta \lambda_{i, K}$ and $\Delta \phi_{i, K}$ denote the changes in the $i^{\text {th }}$ eigenvalue and its corresponding mode shape due to a change in stiffness, respectively. From eigenvalue analysis, presented in equation (1), the following equation can be obtained:

$$
\begin{aligned}
& K^{u} \cdot \Delta \phi_{i, K}-\lambda_{i}^{u} \cdot M^{u} \cdot \Delta \phi_{i, K}+\Delta K \cdot \phi_{i}^{u}-\Delta \lambda_{i, K} \cdot M^{u} \cdot \phi_{i}^{u} \\
& \quad+\Delta K \cdot \Delta \phi_{i, K}-\Delta \lambda_{i, K} \cdot M^{u} \cdot \Delta \phi_{i, K}=0 .
\end{aligned}
$$

This equation can be rearranged by postmultiplying equation (7) by $\left(\Delta \phi_{i, K}\right)^{-1} \cdot \phi_{i}^{u}$, with $\left(\Delta \phi_{i, K}\right)^{-1}$ being the generalized inverse matrix of $\Delta \phi_{i, K}$, and then premultiplying 
equation (7) by $\left(\phi_{i}^{u}\right)^{T}$ and applying the property of orthogonality of mode shape with respect to the mass matrix $\left(\phi_{i}^{u}\right)^{T} \cdot M^{u} \cdot \phi_{i}^{u}=1$; then, equation (7) might be expressed as follows:

$$
\Delta \lambda_{i, K}=\frac{\left(\phi_{i}^{u}\right)^{T} \cdot \Delta K \cdot \phi_{i}^{u}+\left(\phi_{i}^{u}\right)^{T} \cdot \Delta K \cdot \Delta \phi_{i, K}}{\left(\phi_{i}^{u}\right)^{-1} \cdot \Delta \phi_{i, K}+1}
$$

where $\left(\phi_{i}^{u}\right)^{-1}$ refers to the generalized inverse matrix of $\phi_{i}^{u}$.

Similarly, assuming a constant stiffness in the structure, equation (5) might be expressed as follows:

$$
\begin{aligned}
& {\left[K^{u}-\lambda_{i}^{u} \cdot M^{u}-\lambda_{i}^{u} \cdot \Delta M-\Delta \lambda_{i, M} \cdot M^{u}-\Delta \lambda_{i, M} \cdot \Delta M\right]} \\
& \cdot\left(\phi_{i}^{u}+\Delta \phi_{i, M}\right)=0
\end{aligned}
$$

where $\Delta \lambda_{i, M}$ and $\Delta \phi_{i, M}$ denote the changes in the $i^{\text {th }}$ eigenvalue and its corresponding mode shape due to the change in mass, respectively. After applying the eigenvalue analysis presented in equation (1) and operating with the terms in equation (9) the term increment of $\Delta \lambda_{i, M}$ can be written as follows:

$$
\Delta \lambda_{i, M}=\frac{-\lambda_{i}^{u} \cdot\left(\phi_{i}^{u}\right)^{T} \cdot \Delta M \cdot \phi_{i}^{u}\left(\Delta \phi_{i, M}\right)^{-1} \cdot \phi_{i}^{u}-\lambda_{i}^{u} \cdot\left(\phi_{i}^{u}\right)^{T} \cdot \Delta M \cdot \phi_{i}^{u}}{\left(\Delta \phi_{i, M}\right)^{-1} \cdot \phi_{i}^{u}+1+\left(\phi_{i}^{u}\right)^{T} \cdot \Delta M \cdot \phi_{i}^{u}\left(\Delta \phi_{i, M}\right)^{-1} \cdot \phi_{i}^{u}+\left(\phi_{i}^{u}\right)^{T} \cdot \Delta M \cdot \phi_{i}^{u}}
$$

where $\left(\Delta \phi_{i, M}\right)^{-1}$ refers to the generalized inverse matrix of $\Delta \phi_{i, M}$.

When the change in the mode shape is neglected, that is to say the increment of $\Delta \phi_{i, M}$ is equal to zero, equation (10) might be approximated as presented in equation (11). It is important to notice that in this equation, the second order changes in the above equation are neglected.

$$
\Delta \lambda_{i, M} \approx \frac{-\lambda_{i}^{u} \cdot\left(\phi_{i}^{u}\right)^{T} \cdot \Delta M \cdot \phi_{i}^{u}}{1+\left(\phi_{i}^{u}\right)^{-1} \cdot \Delta \phi_{i, M}+\left(\phi_{i}^{u}\right)^{T} \cdot \Delta M \cdot \phi_{i}^{u}} .
$$

From equation (11), we can derive to the following expression:

$$
\left(\phi_{i}^{u}\right)^{T} \cdot \Delta M \cdot \phi_{i}^{u}=-\frac{\Delta \lambda_{i, M}+\Delta \lambda_{i, M} \cdot\left(\phi_{i}^{u}\right)^{-1} \cdot \Delta \phi_{i, M}}{\lambda_{i}^{u}+\Delta \lambda_{i, M}} .
$$

Therefore, equation (11) might be expressed as follows:

$$
\Delta \lambda_{i, M}=\frac{\lambda_{i}^{u} \cdot \Delta \lambda_{i, M}\left(\Delta \phi_{i, M}\right)^{-1} \cdot \phi_{i}^{u}+2 \lambda_{i}^{u} \cdot \Delta \lambda_{i, M}+\lambda_{i}^{u} \cdot \Delta \lambda_{i, M} \cdot\left(\phi_{i}^{u}\right)^{-1} \cdot \Delta \phi_{i, M}}{\lambda_{i}^{u} \cdot\left(\Delta \phi_{i, M}\right)^{-1} \cdot \phi_{i}^{u}+\lambda_{i}^{u}-\Delta \lambda_{i, M}-\Delta \lambda_{i, M} \cdot\left(\phi_{i}^{u}\right)^{-1} \cdot \Delta \phi_{i, M}}
$$

The existence of structural damage and the change in structural mass in a structural system leads to the modification of the vibration modes. These modifications are reflected as changes in modal parameters such as natural frequency, mode shape, and modal damping value. In this study, it is assumed that the changes in eigenvalues due to stiffness perturbations are equivalent to those produced by mass perturbations. Mathematically this can be expressed by joining equations (8) and (13) as follows:

$$
\frac{\left(\phi_{i}^{u}\right)^{T} \cdot \Delta K \cdot \phi_{i}^{u}+\left(\phi_{i}^{u}\right)^{T} \cdot \Delta K \cdot \Delta \phi_{i, K}}{\left(\phi_{i}^{u}\right)^{-1} \cdot \Delta \phi_{i, K}+1}=\frac{\lambda_{i}^{u} \cdot \Delta \lambda_{i, M}\left(\Delta \phi_{i, M}\right)^{-1} \cdot \phi_{i}^{u}+2 \lambda_{i}^{u} \cdot \Delta \lambda_{i, M}+\lambda_{i}^{u} \cdot \Delta \lambda_{i, M} \cdot\left(\phi_{i}^{u}\right)^{-1} \cdot \Delta \phi_{i, M}}{\lambda_{i}^{u} \cdot\left(\Delta \phi_{i, M}\right)^{-1} \cdot \phi_{i}^{u}+\lambda_{i}^{u}-\Delta \lambda_{i, M}-\Delta \lambda_{i, M} \cdot\left(\phi_{i}^{u}\right)^{-1} \cdot \Delta \phi_{i, M}} .
$$

According to the included mode shape (IMS) changes method [32], the changes of the natural frequencies might be analyzed by including the change of mode shapes as
$\Delta \lambda_{i, K}=\left(\phi_{i}^{u}\right)^{T} \cdot \Delta K \cdot \phi_{i}^{u}+\left(\phi_{i}^{u}\right)^{T} \cdot \Delta K \cdot \Delta \phi_{i, K}$. In this way, the following equation can be obtained from the mass and stiffness perturbation method:

$$
\Delta \phi_{i, K}=\frac{\lambda_{i}^{u} \cdot\left(\Delta \lambda_{i, K}-\Delta \lambda_{i, M}\right) \cdot \phi_{i}^{u} \cdot\left(\Delta \phi_{i, M}\right)^{-1} \cdot \phi_{i}^{u}+\Delta \lambda_{i, K} \cdot \lambda_{i}^{u} \cdot \phi_{i}^{u}-\Delta \lambda_{i, K} \cdot \Delta \lambda_{i, M} \cdot \phi_{i}^{u}-2 \lambda_{i}^{u} \cdot \Delta \lambda_{i, M} \cdot \phi_{i}^{u}-\Delta \lambda_{i, M} \cdot\left(\Delta \lambda_{i, K}+\lambda_{i}^{u}\right) \cdot \Delta \phi_{i, M}}{\lambda_{i}^{u} \cdot \Delta \lambda_{i, M}\left(\Delta \phi_{i, M}\right)^{-1} \cdot \phi_{i}^{u}+2 \lambda_{i}^{u} \cdot \Delta \lambda_{i, M}+\lambda_{i}^{u} \cdot \Delta \lambda_{i, M} \cdot\left(\phi_{i}^{u}\right)^{-1} \cdot \Delta \phi_{i, M}} .
$$


In equation (15), the change in mode shape due to stiffness perturbation is expressed as a function of the change in mode shape due to mass perturbation. This equation represents the foundation of the proposed MSCP method, and it demonstrates that the results do not depend on the changes in mass $\Delta M$.

The MSCP method is based on the following four parameters: (i) the change in the $i^{\text {th }}$ frequency $\Delta \lambda_{i, M}$ due to mass perturbation, (ii) the change in the mode shape $\Delta \phi_{i, M}$ due to mass perturbation, (iii) the change in the $i^{\text {th }}$ mode shape $\Delta \lambda_{i, K}$ due to stiffness perturbation, and (iv) the $i^{\text {th }}$ frequency of the intact structure $\lambda_{i}^{u}$. The mass perturbation model can be calculated by adding a vehicle load at different locations. In this way, both the frequency and its corresponding mode shape of the mass perturbation structure can be also obtained from dynamic testing. The changes in mode shape due to structural damage can be estimated with equation (15).

In order to obtain the mode shapes in the MSCP method, modal parameters, such as natural frequencies and mode shapes of the intact structure and mass perturbation structure, should be obtained through dynamic testing. The main advantage of the MSCP method compared with other alternatives in the literature is that it only requires to monitor the frequency of damaged structures. Thanks to this characteristic, the damage diagnosis can be performed with high efficiency and reduced monitoring cost. The main problem for the application of the MSCP method is that a suitable mass perturbation model should be selected in advance to calculate the mode shapes of the damaged structures. For this purpose, an objective function must be established to select the optimal mass perturbation model. The definition of this function is presented in the following section.

\subsection{Objective Function for Selecting the Mass Perturbation} Model. The change in natural frequency varies directly with the square root of the change in stiffness and inversely with the square root of the change in mass. The same changes in natural frequency are produced by a change in stiffness or mass $[37,38]$. Both stiffness and mass perturbation modify the modal parameters of the structures. This leads to the similar frequency principle, which states that the changes in a natural frequency produced by mass and stiffness perturbations are equivalent. According to this principle, an objective function for selecting the mass perturbation model might be expressed as follows:

$$
F_{\text {obj }}=\min \left|\frac{\left(\lambda_{i, K}-\lambda_{i, M}\right)}{\lambda_{i, K}}\right|,
$$

where $\lambda_{i, K}$ and $\lambda_{i, M}$ denote the $i^{\text {th }}$ frequency due to stiffness and mass perturbation, respectively.

The three steps used by the MSCP method to monitor the mode shapes of damaged structures are as follows: Firstly, the modal parameters due to mass perturbation are measured and the natural frequencies are monitored. Secondly, the similar principle between the frequency due to mass perturbation and the frequency due to damage is optimized to select an adequate mass perturbation model. Thirdly, the optimum mass perturbation model is used to calculate the mode shapes due to damage using the MSCP method (see equation (15)).

\section{Numerical and Experimental Investigations}

3.1. Numerical Model and Experimental Setup. A simply supported reinforced concrete beam with a length of $3.2 \mathrm{~m}$ and a rectangular cross section of $0.12 \times 0.15 \mathrm{~m}$ was used to investigate the effectiveness to identify mode shapes of the MSCP method. The numerical model and the laboratory tests were established accordingly. A two-dimensional simply supported beam was modeled numerically using a FEM with an Euler Bernoulli beam modeled with MATLAB software. The density, modulus of elasticity, and Poisson's ratio are taken as $2500 \mathrm{~kg} / \mathrm{m}^{3}, 3.0 \times 10^{4} \mathrm{MPa}$, and 0.3 , respectively. The beam structure was divided into 21 nodes and 20 beam elements as presented in Figure 1.

In the laboratory test, a reinforced concrete (RC) beam was placed on two concrete abutments as presented in Figure 2. The dimensions of both the beam and the abutments are included in this figure. In order to measure the modal parameters of mass perturbation structures, the 20 mass perturbation locations presented in this figure were considered. The length of each mass perturbation location is assumed as $0.15 \mathrm{~m}$. The instruments used include an oscilloscope, a charge optimal modulator, a piezoelectric accelerometer, a computer, and signal cables. Seven piezoelectric accelerometers were connected to the oscilloscope to capture the acceleration signals in real time. The location of these sensors is presented in Figure 2. The output of the piezoelectric accelerometer and the load input were amplified and filtered using a charge optimal modulator. The ambient vibration method was adopted in the laboratory test $[39,40]$. The sample frequency was selected as $2000 \mathrm{~Hz}$. The natural frequencies and mode shapes were obtained using the fast Fourier transform and cross-correlation function analysis methods [41].

A picture of the real modal test including all the used instruments is presented in Figure 3.

\subsection{Mass Perturbation Scenarios and Damage Scenarios.} To obtain mass perturbation scenarios in the FEM, an $80 \%$ mass perturbation was added from elements 1 to 20 . These scenarios are defined as mass perturbations M1 to M20, respectively. In the laboratory test, the location of the mass perturbation location corresponds with that presented in Figure 2. A concrete specimen weighting $5.65 \mathrm{~kg}$, about 5.9 percentage of the test beam, is placed on the RC test beam from mass perturbation location 1 to 20, respectively. These scenarios are named mass perturbations T-M1 to T-M20.

The FEM and experimental RC test beam were divided into four damage scenarios, respectively: (a) In the FEM, the damage was simulated by the element stiffness decrease at element 10 . The damage severity was assumed as $5 \%, 10 \%$, $15 \%$, and $20 \%$. These damage scenarios are named $1(\mathrm{D}-1)$, 2(D-2), 3(D-3), and 4(D-4), respectively; (b) In the RC test beam, the damage scenarios were simulated with cracks at the $1 / 4$ and $1 / 2$ beam length locations as shown in Figure 3. 


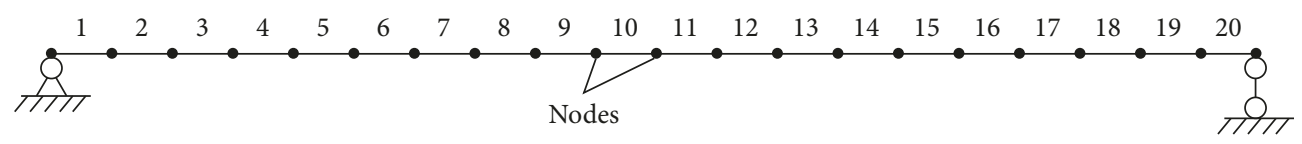

FIGURE 1: Details of the simply supported beam FEM.

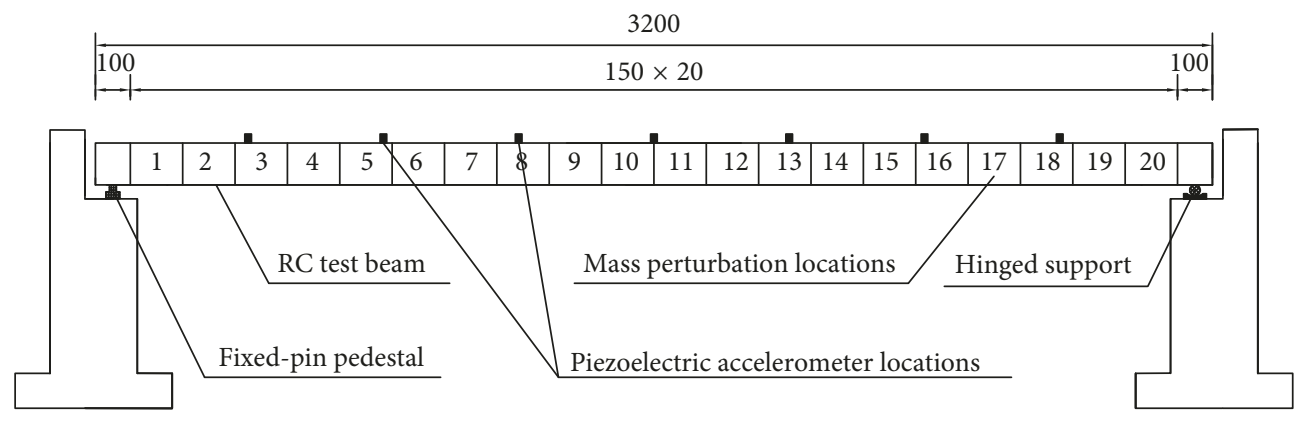

Figure 2: Detailed layout of the experimental setup (units: mm).

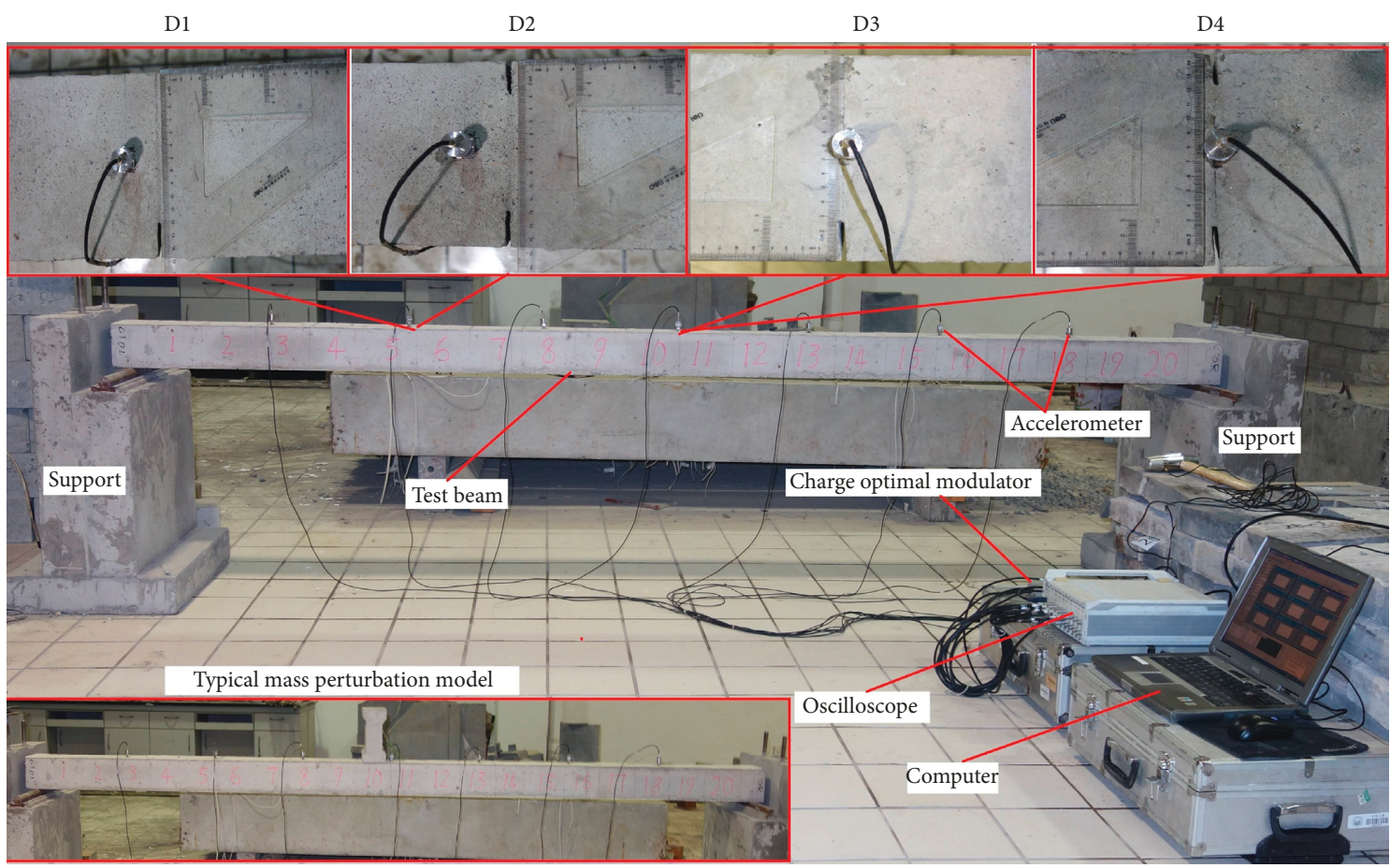

Figure 3: Modal test for RC test beam.

These cracks had a length of $25 \mathrm{~mm}$ and a width of $2 \mathrm{~mm}$. The locations of the cracks (D1 to D4) of each damage scenario are listed in Table 1.

When a structure is subjected to a change in mass or stiffness, the dynamic parameters, such as natural frequency and mode shape, will change consequently. To evaluate this phenomenon, the following two steps are followed in the laboratory test. Firstly, the additional concrete specimen mass $(5.65 \mathrm{~kg})$ was added to the intact $\mathrm{RC}$ beam from mass perturbation locations 1 to 20. Thus, the mass perturbation scenarios from T-M1 to T-M20 were obtained and the modal parameters were measured. Secondly, the stiffness damage scenarios were simulated by cutting the cracks specified in Table 1. In this way, the damage scenarios from D1 to D4 were obtained and the modal parameters were measured. In the FEM analysis, the FEM updates are obtained according to the similar frequency principle with the laboratory RC beam. The natural frequencies of the FEM analysis and the experimental test are presented in Table 2. Because of the many factors influencing the experimental test process, the measured second and fourth frequencies in case D4 are larger than those in case D3. These results are not 
TABLe 1: Crack number and location of each damage scenario.

\begin{tabular}{lcc}
\hline \multirow{2}{*}{ Damage scenarios } & \multicolumn{2}{c}{ Crack locations } \\
& $1 / 4$ beam length & $1 / 2$ beam length \\
\hline D1 & 1 & None \\
D2 & 2 & None \\
D3 & 2 & 1 \\
D4 & 2 & 2 \\
\hline
\end{tabular}

consistent with the tendency of the frequencies to decrease with damage severity. This result can be explained by the following two reasons. (1) The difference between case D3 and case D4 refers to the damage severity in midspan. This point corresponds with the zero-point location of the second and fourth modes, and for this reason, the influence on the frequency change is small. The FEM analysis proved that the change rate of the first four frequencies are $-0.39 \%,-0.01 \%$, $-0.33 \%$, and $-0.01 \%$, respectively. The changes of the second and fourth frequencies are masked by the environmental noise and processing errors easily in the laboratory test. (2) The change rate of the first four frequencies obtained by the experimental test are $-0.12 \%, 0.34 \%,-0.73 \%$, and $0.09 \%$, respectively, and the average is $-0.11 \%$. These results demonstrate that the damage severity of case D4 is more serious than in case D3. The results of the experimental test are properly related with the damage scenarios in Table 1.

3.3. Optimum for Mass Perturbation Model. The second step of the MSCP method to calculate the mode shapes is to select the optimum mass perturbation model through the objective function in equation (16). The obtained optimization mass perturbation locations of the damage scenarios are shown in Table 3. This table shows that the damage scenarios between the FEM and the laboratory RC test beam are not exactly the same. It is difficult to find a consistent correspondence between the damage scenarios of the FEM and the RC test beam. To do so, only the results of FEM and laboratory RC test beam can be analyzed.

Table 3 demonstrates that different mode numbers have different optimization mass perturbation locations. From these results, it can be concluded that the mass perturbation element location of each mode shape order is different for the same damage scenario. This is mainly due to the fact that the damage severity is different, and as illustrated in (see equation (15)) the MSCP method it is independent of changes in mass $\Delta M$. Therefore, the mass change does not need to be considered by the MSCP method. Although the mass changes should generate different optimum results using equation (16), the mode shape calculation results of the damaged structures are not affected, and the same results are always obtained.

3.3.1. Results for Mode Shape Identification. From the above analysis it can be concluded that one of the most evident advantages of the MSCP method is that it does not require the identification of the mode shapes of the damaged structure. Therefore, the monitoring cost could be
TABLE 2: Natural frequencies of the FEM analysis and the laboratory test $(\mathrm{Hz})$.

\begin{tabular}{cccccc}
\hline \multirow{2}{*}{ Scenarios } & & \multicolumn{4}{c}{ Mode no. } \\
& & First & Second & Third & Fourth \\
\hline \multirow{4}{*}{ FEM analysis } & Intact & 19.461 & 76.727 & 167.245 & 275.853 \\
& D-1 & 19.405 & 76.720 & 166.825 & 275.830 \\
& D-2 & 19.344 & 76.712 & 166.367 & 275.805 \\
& D-3 & 19.275 & 76.704 & 165.866 & 275.777 \\
& D-4 & 19.200 & 76.695 & 165.314 & 275.745 \\
\hline \multirow{5}{*}{ Laboratory test $t$ Intact } & 19.461 & 76.575 & 165.648 & 281.351 \\
& D1 & 19.312 & 75.951 & 162.699 & 280.012 \\
& D2 & 19.180 & 75.601 & 161.789 & 279.330 \\
& D3 & 19.111 & 75.259 & 161.466 & 278.582 \\
& D4 & 19.089 & 75.518 & 160.288 & 278.823 \\
\hline
\end{tabular}

TABle 3: Optimization mass perturbation location of damage scenarios.

\begin{tabular}{lccccc}
\hline \multirow{2}{*}{ Damage scenarios } & \multicolumn{4}{c}{ Mode no. } \\
& & First & Second & Third & Fourth \\
\hline \multirow{4}{*}{ FEM analysis } & D-1 & 17 & 10 & 7 & 15 \\
& D-2 & 3 & 10 & 19 & 15 \\
& D-3 & 16 & 10 & 12 & 15 \\
& D-4 & 4 & 10 & 14 & 15 \\
\hline \multirow{5}{*}{ Laboratory test } & D1 & 3 & 19 & 6 & 17 \\
& D2 & 3 & 3 & 14 & 16 \\
& D3 & 4 & 14 & 6 & 16 \\
& D4 & 5 & 6 & 14 & 11 \\
\hline
\end{tabular}

decreased. In order to identify the damage, the intact and damaged modal parameters should be measured and identified by using ambient vibration methods. Unfortunately, these two states (intact and undamaged) cannot be measured at the same time as they are separated by years. Another inconvenience from these procedures is the existence of a number of errors in both the intact and the damaged measurement processes, in particular errors in the mode shapes, that complicate the damage identification.

To validate the effectiveness of the MSCP method the mode shapes obtained by the FEM were analyzed. The first four mode shapes from the actual damaged mode shapes of the FEM, considering damage scenario D- 1 of $5 \%$ error, and from the MSCP method are shown in Figure 4.

Figure 4 demonstrates that the mode shapes obtained using the MSCP method are more precise than those obtained by the monitoring and identifying method with a damage scenario D-1 of $5 \%$ error. This proves the effectiveness of the MSCP method. In the laboratory test, the mode shapes from actual damage mode shapes of the test and from the MSCP method are compared in Figure 5.

3.3.2. Modal Assurance Criterion (MAC). For further comparison, the Modal Assurance Criterion (MAC) method is discussed for both the FEM simulation and the laboratory test [42]. The mode shapes for the damaged 


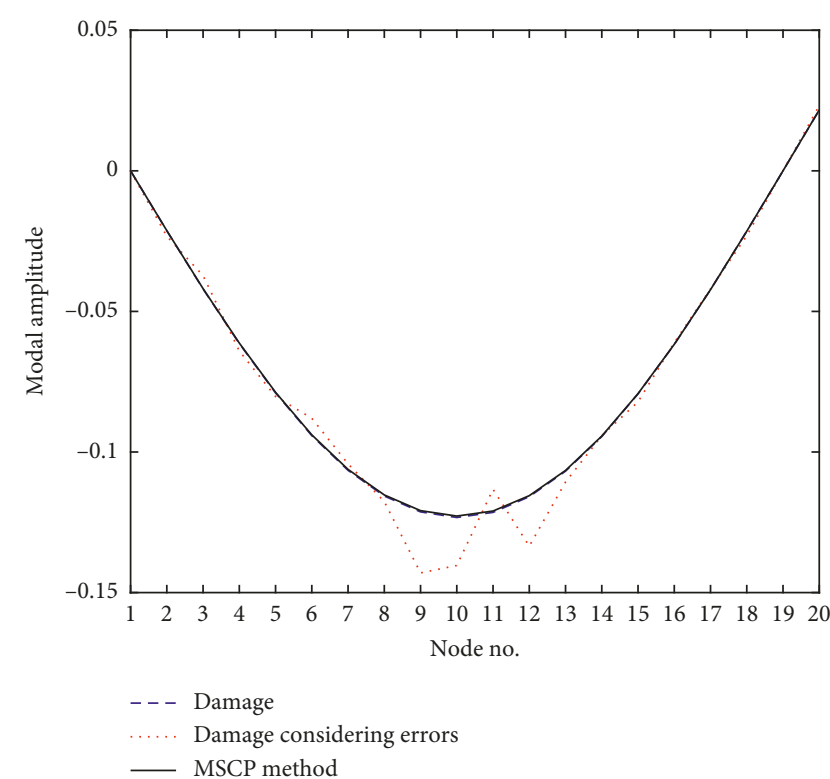

(a)

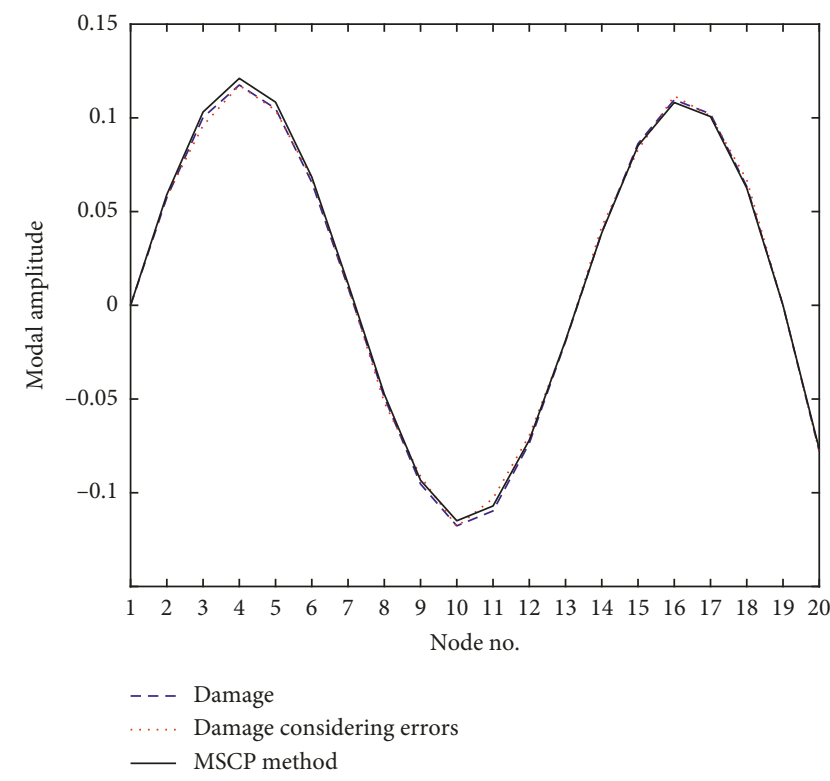

(c)

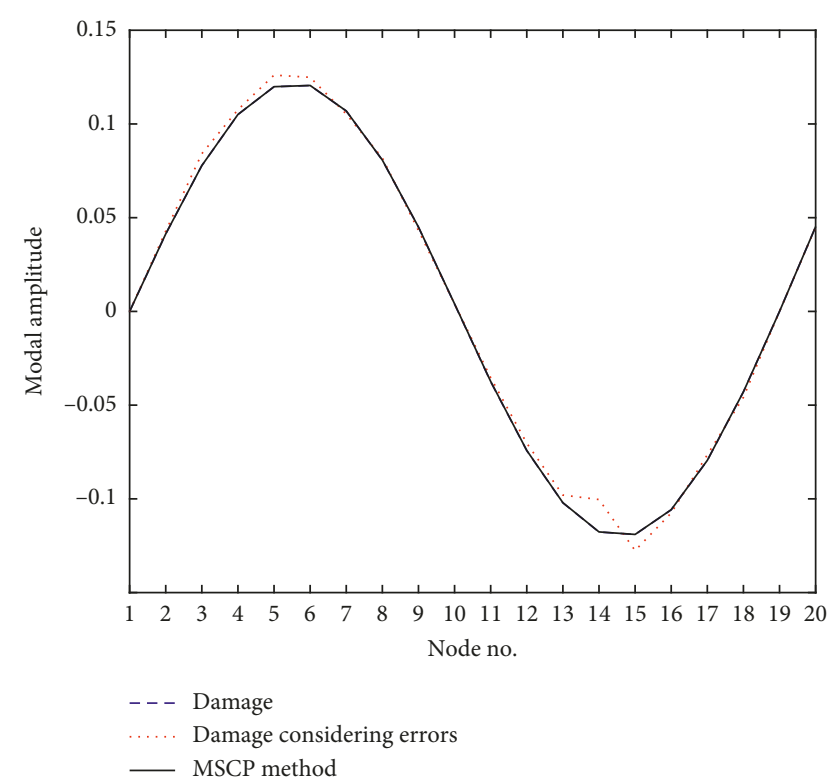

(b)

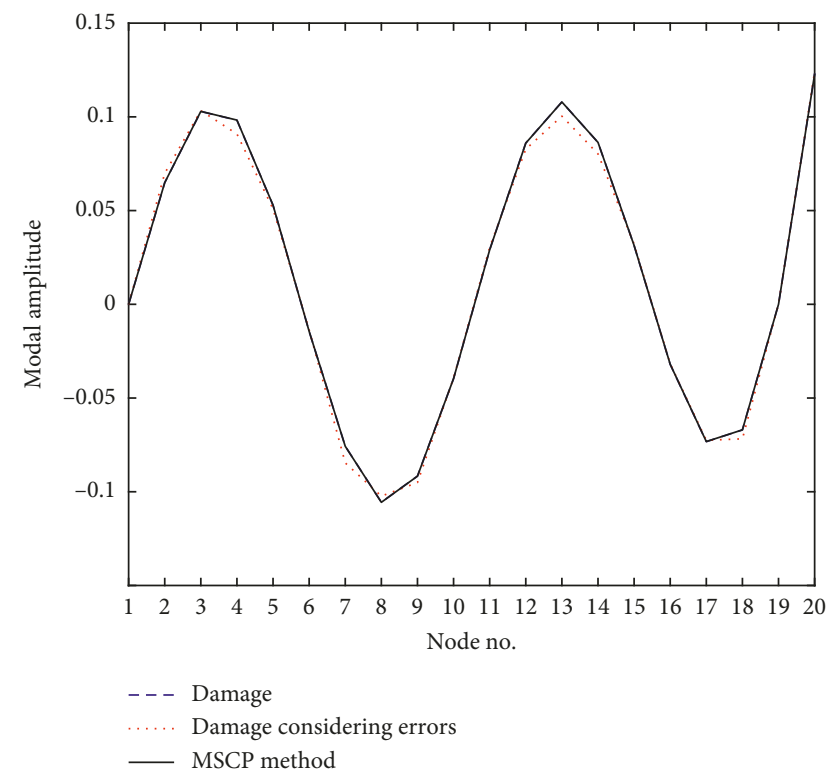

(d)

Figure 4: Typical mode shapes (D-1): (a) first mode, (b) second mode, (c) third mode, and (d) fourth mode.

structure and the MSCP method can be verified using the MAC, as follows:

$$
\operatorname{MAC}(M, i)=\frac{\left|\phi_{M}^{T} \cdot \phi_{i}^{d}\right|^{2}}{\left|\phi_{M}^{T} \cdot \phi_{M}\right|\left|\phi_{i}^{d T} \cdot \phi_{i}^{d}\right|},
$$

where $\phi_{M}$ and $\phi_{i}^{d}$ are the mode shapes due to mass perturbation and damaged mode shapes, respectively. The highest $\operatorname{MAC}(M, i)$ factors indicate the most likely pairings of the damaged mode $i$ and mass perturbation mode $M$.
The correlation between the mode shapes of the damaged structure and the mass perturbation structures is checked using the MAC factors. The MAC factors obtained by the MSCP method for different damage scenarios of the FEM analysis (D-1 to D-4) and the laboratory tests (D1 to D4) are shown in Table 4.

Table 4 shows high MAC values of mode shapes for the MSCP method compared to damaged structures. This demonstrates that the calculated damaged mode shapes using the MSCP method are more accurate than traditional recognition methods. In order to compare the precision of 


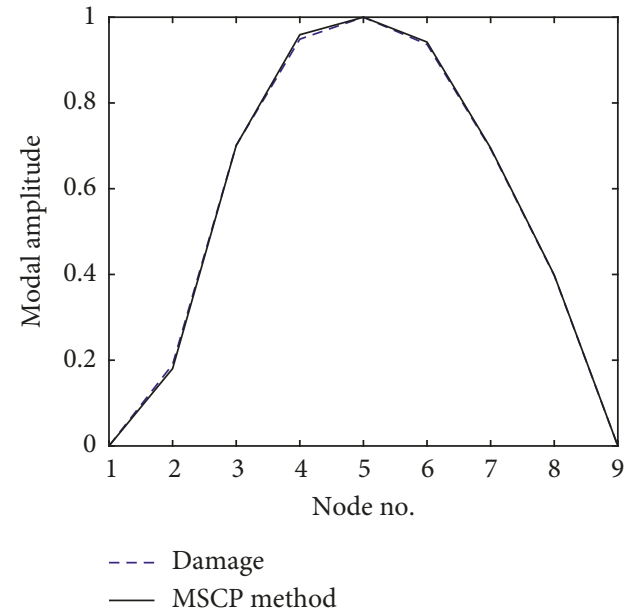

(a)

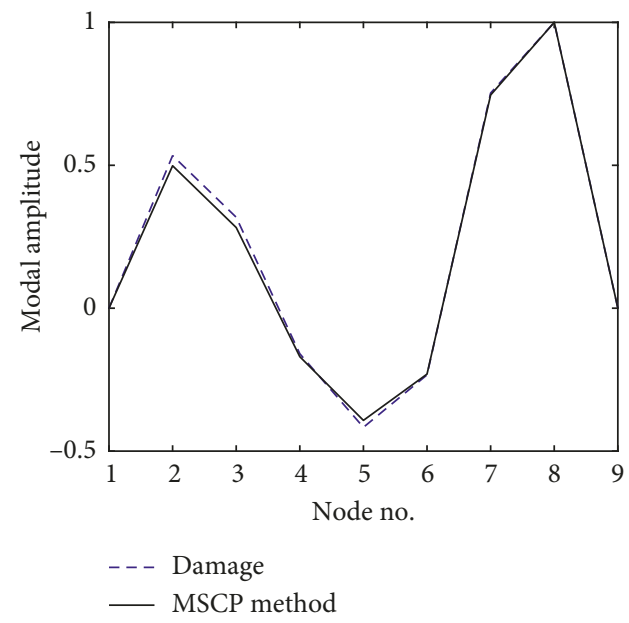

(c)

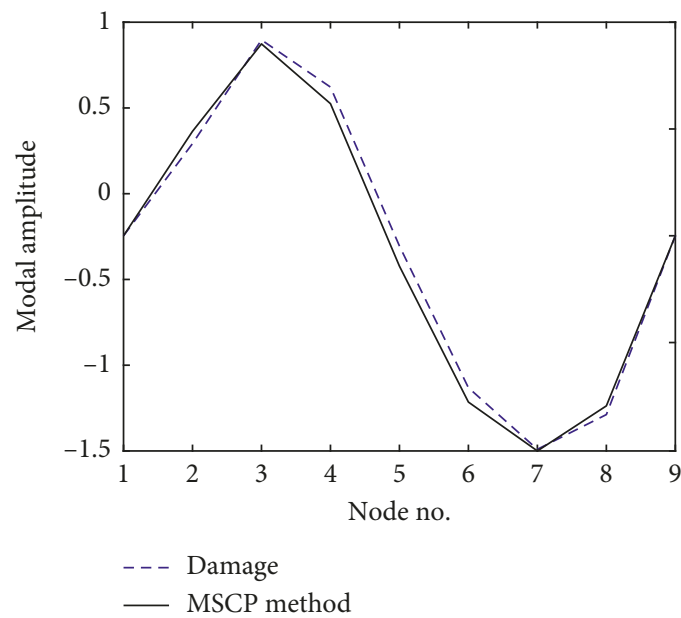

(b)

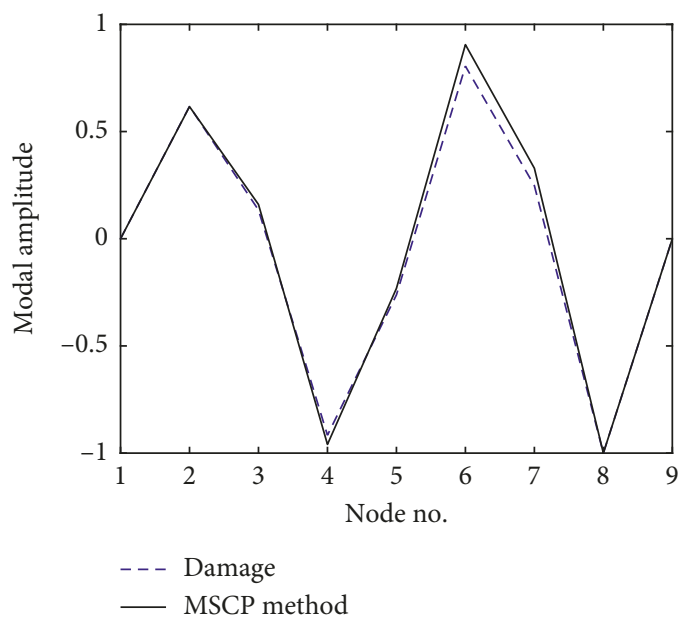

(d)

Figure 5: Typical mode shapes (D1): (a) first mode, (b) second mode, (c) third mode, and (d) fourth mode.

TABLE 4: MAC factors obtained by the MSCP method for different damaged scenarios in the FEM analysis and the laboratory tests.

\begin{tabular}{|c|c|c|c|c|c|c|}
\hline \multirow{2}{*}{\multicolumn{2}{|c|}{ Damage scenarios }} & \multicolumn{5}{|c|}{ MSCP method } \\
\hline & & Mode no. & First & Second & Third & Fourth \\
\hline \multirow{16}{*}{ FEM analysis } & \multirow{4}{*}{ D-1 } & First & 1.0000 & 0.0003 & 0.0008 & 0.0019 \\
\hline & & Second & 0.0003 & 1.0000 & 0.0034 & 0.0080 \\
\hline & & Third & 0.0007 & 0.0033 & 1.0000 & 0.0234 \\
\hline & & Fourth & 0.0019 & 0.0080 & 0.0232 & 1.0000 \\
\hline & \multirow{5}{*}{ D-2 } & First & 1.0000 & 0.0003 & 0.0008 & 0.0019 \\
\hline & & Second & 0.0003 & 1.0000 & 0.0034 & 0.0080 \\
\hline & & Third & 0.0007 & 0.0033 & 1.0000 & 0.0235 \\
\hline & & Fourth & 0.0018 & 0.0080 & 0.0230 & 1.0000 \\
\hline & & First & 1.0000 & 0.0002 & 0.0009 & 0.0018 \\
\hline & \multirow{3}{*}{ D-3 } & Second & 0.0003 & 1.0000 & 0.0035 & 0.0080 \\
\hline & & Third & 0.0007 & 0.0032 & 0.9999 & 0.0236 \\
\hline & & Fourth & 0.0018 & 0.0079 & 0.0228 & 1.0000 \\
\hline & \multirow{4}{*}{ D-4 } & First & 1.0000 & 0.0002 & 0.0009 & 0.0018 \\
\hline & & Second & 0.0003 & 1.0000 & 0.0035 & 0.0080 \\
\hline & & Third & 0.0006 & 0.0031 & 0.9998 & 0.0237 \\
\hline & & Fourth & 0.0018 & 0.0079 & 0.0226 & 1.0000 \\
\hline
\end{tabular}


TABLE 4: Continued.

\begin{tabular}{|c|c|c|c|c|c|c|}
\hline \multirow{2}{*}{\multicolumn{2}{|c|}{ Damage scenarios }} & \multicolumn{5}{|c|}{ MSCP method } \\
\hline & & Mode no. & First & Second & Third & Fourth \\
\hline \multirow{16}{*}{ Laboratory test } & \multirow{4}{*}{ D1 } & First & 1.0000 & 0.0032 & 0.0029 & 0.0011 \\
\hline & & Second & 0.0045 & 0.9994 & 0.0830 & 0.0214 \\
\hline & & Third & 0.0113 & 0.0568 & 0.9909 & 0.0065 \\
\hline & & Fourth & 0.0090 & 0.0269 & 0.0246 & 0.9679 \\
\hline & \multirow{5}{*}{ D2 } & First & 1.0000 & 0.0046 & 0.0066 & 0.0055 \\
\hline & & Second & 0.0045 & 0.9995 & 0.0253 & 0.0225 \\
\hline & & Third & 0.0114 & 0.0571 & 0.9907 & 0.0166 \\
\hline & & Fourth & 0.0091 & 0.0258 & 0.0539 & 0.9968 \\
\hline & & First & 1.0000 & 0.0082 & 0.0047 & 0.0044 \\
\hline & \multirow{3}{*}{ D3 } & Second & 0.0043 & 0.9995 & 0.0376 & 0.0284 \\
\hline & & Third & 0.0115 & 0.0503 & 0.9950 & 0.0094 \\
\hline & & Fourth & 0.0091 & 0.0252 & 0.0451 & 0.9939 \\
\hline & \multirow{4}{*}{ D4 } & First & 0.9999 & 0.0055 & 0.0047 & 0.0068 \\
\hline & & Second & 0.0038 & 0.9999 & 0.0292 & 0.0248 \\
\hline & & Third & 0.0118 & 0.0509 & 0.9940 & 0.0151 \\
\hline & & Fourth & 0.0098 & 0.0260 & 0.0409 & 0.9976 \\
\hline
\end{tabular}

the damaged mode shapes obtained with the MSCP method with those of the monitoring and identifying method, the MAC values of damaged mode shapes with $5 \%$ error and those of actual damaged mode shapes were calculated. The comparison results of the MAC values in the FEM and in the laboratory tests are shown in Figures 6 and 7 , respectively.

Figure 6 demonstrates that the MAC values of the damaged mode shapes obtained with the MSCP method are higher than those of the damaged structure with a 5\% error in the FEM (damage scenario 1, defined as D-1). This proves the higher precision of the MSCP method. The results of the other damage scenarios (D-2, D-3, and D-4) confirm the same results with the exception of the third order mode shapes in damage scenario 4 (D-4). The main reason for this phenomenon is the severity of damage scenario $4(\mathrm{D}-4)$ is the highest (20\%).

Figure 7 shows that the MAC values of the damaged mode shapes obtained using the MSCP method are higher than those of the measurement damaged structure mode shapes in damage scenario 1 (D1). The highest MAC values indicate the most likely pairings of the comparing mode shapes. The minimum of MAC factors of the MSCP method with damage and intact with damage is 0.999 and 0.993 , respectively. Therefore, it can be concluded that the damaged mode shapes obtained using the MSCP method are very precise.

The obtained results of the MSCP method are more precise against lower damage severities by FEM analysis. This indicates that the proposed MSCP method is more suitable for minor damage scenarios.

\section{Case Study Application}

4.1. Bridge Description. A three-dimensional global FEM of the Wangyu River Bridge, which is located across the Wangyu River, between Wuxi and Suzhou in China, is analyzed using the general purpose commercial software ANSYS as illustrated in Figure 8. The main spans of this bridge are designed as four-span prestressed concrete (PC) continuous box

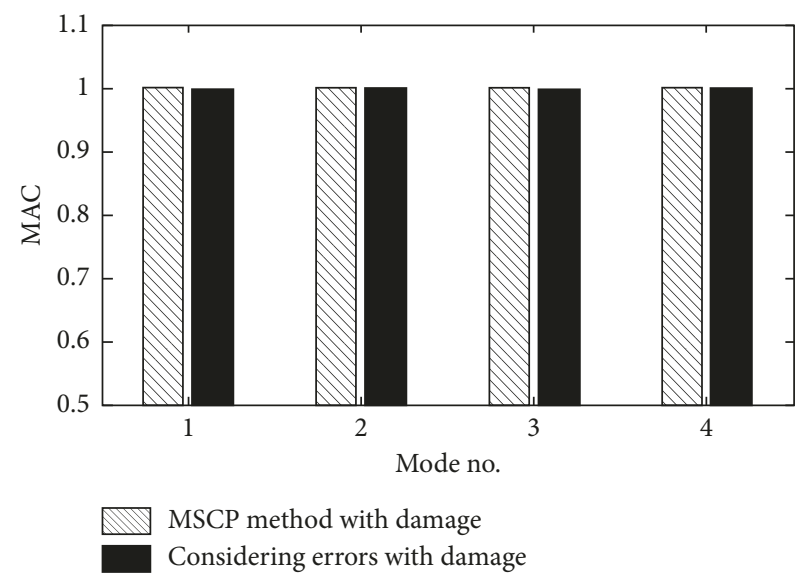

FIgURE 6: Comparison results of MAC in FEM (D-1).

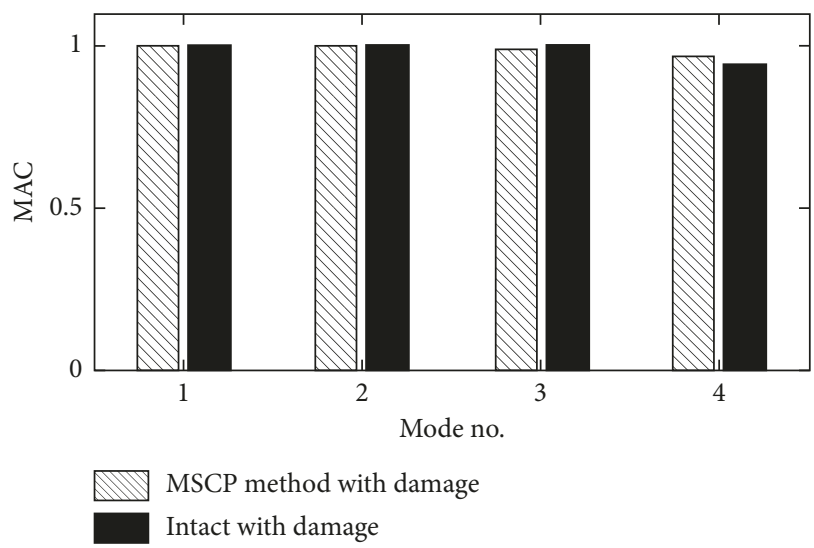

FIgURE 7: Comparison results of MAC in the laboratory test (D1).

girders with the arrangement of $50 m+2 \times 80 m+50 m$. The beam elements in the ANSYS element library are chosen to model the structural components of the bridge. There are 376 nodes and 214 elements in the global FEM. 


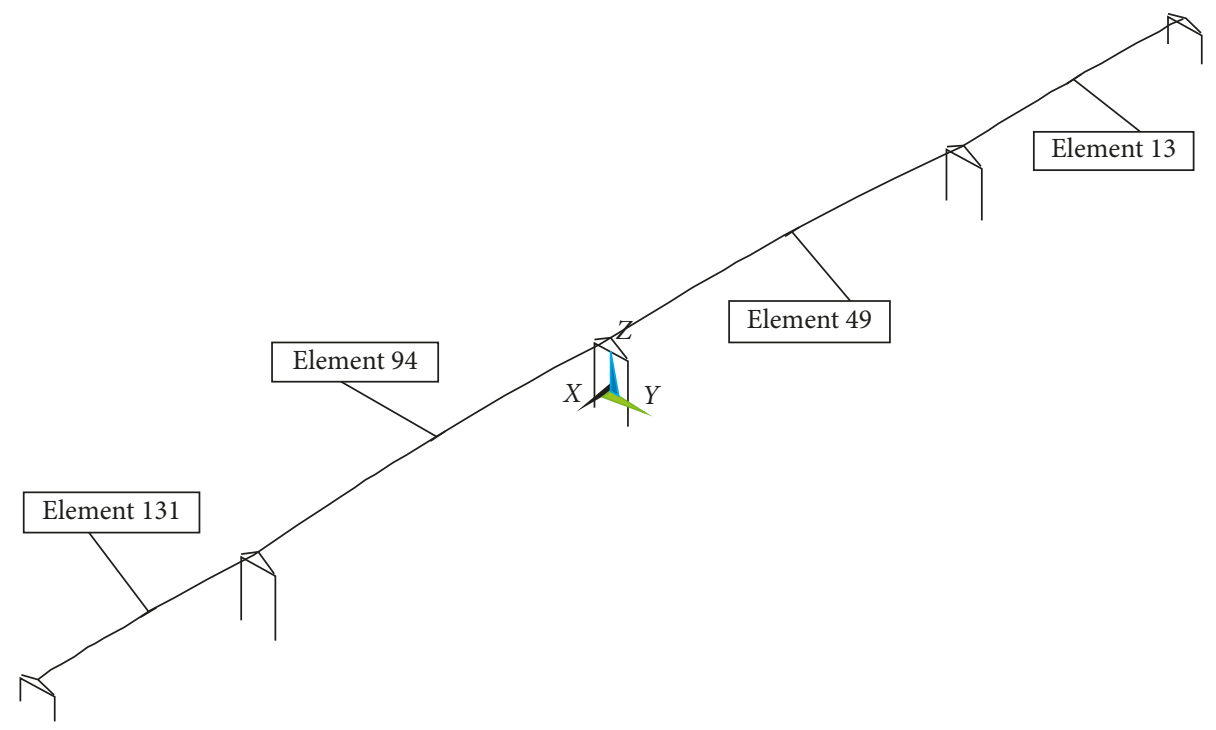

Figure 8: Three-dimensional global FEM of Wangyu River Bridge.

4.2. Mass Perturbation Scenarios and Damage Scenarios. The main box girder of the Wangyu River Bridge was divided into 142 elements. Therefore, a total of 142 mass perturbation models were considered by changing the density of every main box girder element from 2600 to $6000 \mathrm{~kg} / \mathrm{m}^{3}$.

Four damage scenarios are considered on the main box girder. The damaged severity is assumed as $20 \%$, and the damage locations were selected in elements $13,49,94$, and 131 highlighted in Figure 8. These locataions are defined as damaged scenarios I(D-I), II(D-II), III(D-III), and IV(D-IV), respectively. The natural frequencies and mode shapes of the intact structure, mass perturbation scenarios, and damage scenarios were analyzed in the FEM. The first six orders of frequency are shown in Table 5.

\subsection{Practical Application}

4.3.1. Optimum for Mass Perturbation Model. The optimum for the mass perturbation model was selected through the objective function (equation (16)), and the obtained results are shown in Table 6.

Table 6 shows no correlation for the mass perturbation location of the damage scenarios. Therefore, it can be concluded that different damage scenarios have different mass perturbation models and again that the MSCP method is independent of a change in mass $\Delta M$.

4.3.2. Results for Damaged Structure Mode Shapes. The mode shapes from the damaged structure, damage considering errors, and the MSCP method are shown in Figure 9. This figure illustrates that the mode shapes obtained using the MSCP method are more precise than those obtained by the monitoring and identifying method with a $5 \%$ error. These results also prove again efficiency of the MSCP method.
Table 5: Natural frequencies of Wangyu River Bridge (Hz).

\begin{tabular}{lcccccc}
\hline \multirow{2}{*}{ Scenarios } & \multicolumn{5}{c}{ Mode no. } \\
& First & Second & Third & Fourth & Fifth & Sixth \\
\hline Intact & 1.1147 & 1.7703 & 2.8049 & 3.2438 & 3.4060 & 5.6567 \\
D-I & 1.1133 & 1.7673 & 2.8048 & 3.2429 & 3.4024 & 5.6565 \\
D-II & 1.1136 & 1.7691 & 2.8048 & 3.2415 & 3.4045 & 5.6563 \\
D-III & 1.1135 & 1.7693 & 2.8039 & 3.2428 & 3.4044 & 5.6563 \\
D-IV & 1.1131 & 1.7669 & 2.8047 & 3.2437 & 3.3988 & 5.6558 \\
\hline
\end{tabular}

TABle 6: Optimization mass perturbation location of damage scenarios.

\begin{tabular}{lcccccc}
\hline \multirow{2}{*}{ Damage scenarios } & \multicolumn{7}{c}{ Mode no. } \\
& First & Second & Third & Fourth & Fifth & Sixth \\
\hline D-I & 11 & 59 & 11 & 9 & 100 & 71 \\
D-II & 9 & 24 & 21 & 28 & 77 & 73 \\
D-III & 124 & 113 & 39 & 13 & 24 & 70 \\
D-IV & 15 & 137 & 137 & 80 & 20 & 69 \\
\hline
\end{tabular}

4.3.3. Comparison of Modal Assurance Criterion (MAC). The MAC method is used to check the correlation between mode shapes for the damaged structure and the mass perturbation structure. The MAC values of the MSCP method and the damaged structures of the Wangyu River Bridge for the four damage scenarios (D-I to D-IV) are shown in Table 7. This table shows that high MAC values of mode shapes are obtained by the MSCP method compared to the damaged scenarios. These results demonstrate the higher accuracy of the damaged mode shapes obtained by the MSCP method. The comparison results of the MAC values are shown in Figure 10.

Figure 10 demonstrates that the MAC values of the damage mode shapes obtained using the MSCP method are higher than those of the damaged structure in D-1 (that is considering $5 \%$ error in the FEM). The highest MAC factors indicate the most likely pairings of the comparing mode shapes. 

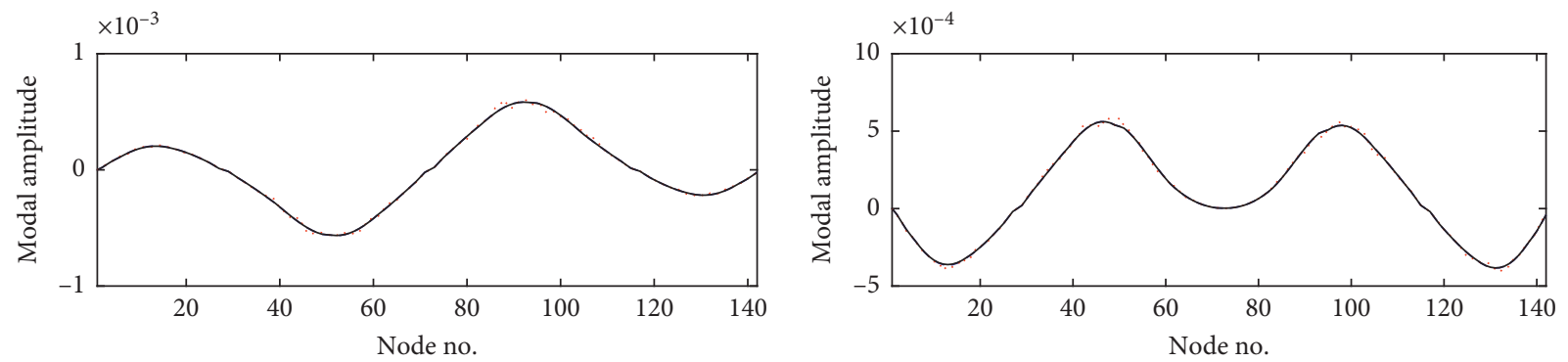

- - - Damage

..... Damage considering errors

- MSCP method

(a)

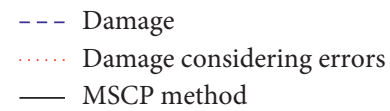

(b)
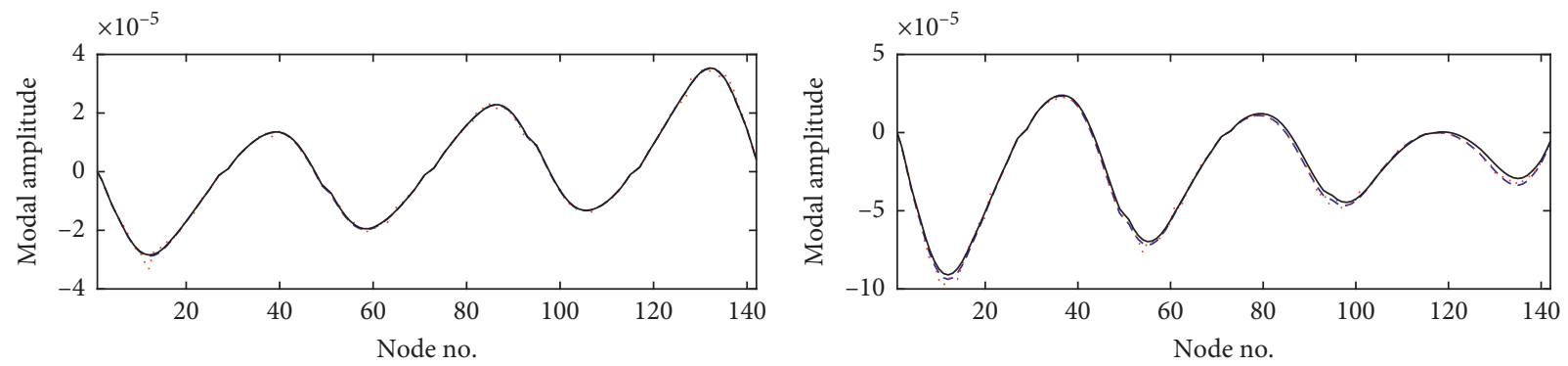

- - - Damage

..... Damage considering errors

— MSCP method

(c)

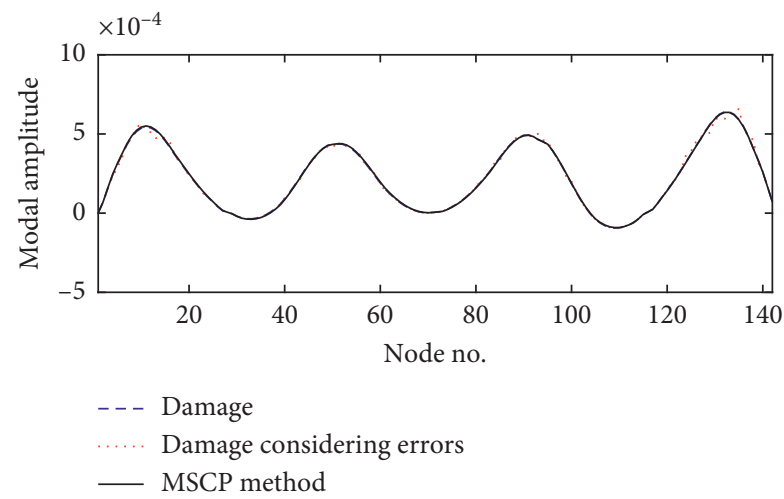

(e)

- - - Damage

Damage considering errors

— MSCP method

(d)

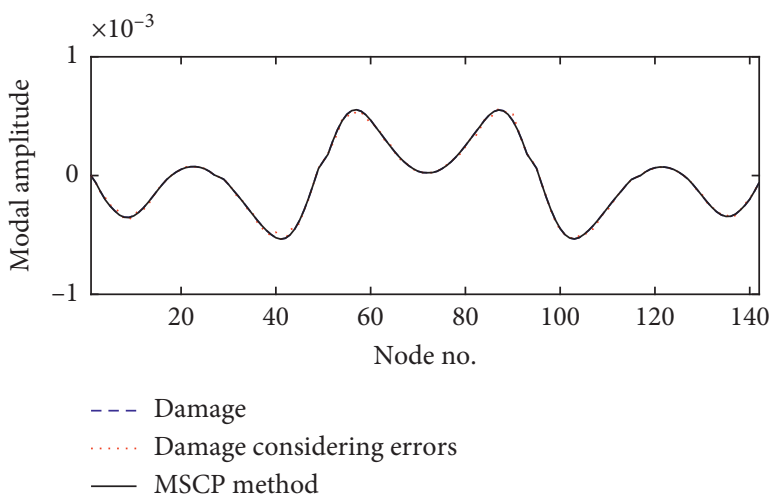

(f)

Figure 9: Typical mode shapes of Wangyu River Bridge (D-I): (a) first mode, (b) second mode, (c) third mode, (d) fourth mode, (e) fifth mode, and (f) sixth mode.

TABLE 7: MAC of MSCP method and damaged structures of the Wangyu River Bridge.

\begin{tabular}{lccccccc}
\hline \multirow{2}{*}{ Damage scenarios } & & \multicolumn{3}{c}{ MSCP method } \\
& Mode no. & First & Second & Third & Fourth & Fifth & 0.0000 \\
& First & 1.0000 & 0.0000 & 0.0007 & 0.0000 & 0.0000 \\
& Second & 0.0000 & 1.0000 & 0.0009 & 0.0070 & 0.0025 & 0.0003 \\
D-I & Third & 0.0005 & 0.0004 & 0.9998 & 0.1681 & 0.0474 & 0.0000 \\
& Fourth & 0.0000 & 0.0058 & 0.2128 & 0.9978 & 0.5704 & 0.0004 \\
& Fifth & 0.0000 & 0.0022 & 0.0381 & 0.6269 & 0.9999 & 0.0001 \\
& Sixth & 0.0000 & 0.0003 & 0.0000 & 0.0004 & 0.0001 & 1.0000 \\
\hline
\end{tabular}


TABLE 7: Continued.

\begin{tabular}{|c|c|c|c|c|c|c|c|}
\hline \multirow{2}{*}{ Damage scenarios } & \multicolumn{7}{|c|}{ MSCP method } \\
\hline & Mode no. & First & Second & Third & Fourth & Fifth & Sixth \\
\hline \multirow{6}{*}{ D-II } & First & 1.0000 & 0.0000 & 0.0006 & 0.0000 & 0.0000 & 0.0000 \\
\hline & Second & 0.0000 & 1.0000 & 0.0006 & 0.0064 & 0.0023 & 0.0003 \\
\hline & Third & 0.0006 & 0.0006 & 1.0000 & 0.2073 & 0.0432 & 0.0000 \\
\hline & Fourth & 0.0000 & 0.0066 & 0.2035 & 1.0000 & 0.5804 & 0.0004 \\
\hline & Fifth & 0.0000 & 0.0024 & 0.0425 & 0.5769 & 1.0000 & 0.0001 \\
\hline & Sixth & 0.0000 & 0.0003 & 0.0000 & 0.0004 & 0.0001 & 1.0000 \\
\hline \multirow{6}{*}{ D-III } & First & 1.0000 & 0.0000 & 0.0006 & 0.0000 & 0.0000 & 0.0000 \\
\hline & Second & 0.0000 & 1.0000 & 0.0006 & 0.0064 & 0.0023 & 0.0003 \\
\hline & Third & 0.0006 & 0.0006 & 1.0000 & 0.2036 & 0.0427 & 0.0000 \\
\hline & Fourth & 0.0000 & 0.0065 & 0.2020 & 1.0000 & 0.5820 & 0.0004 \\
\hline & Fifth & 0.0000 & 0.0024 & 0.0432 & 0.5811 & 1.0000 & 0.0001 \\
\hline & Sixth & 0.0000 & 0.0003 & 0.0000 & 0.0004 & 0.0001 & 1.0000 \\
\hline \multirow{6}{*}{ D-IV } & First & 1.0000 & 0.0000 & 0.0007 & 0.0000 & 0.0000 & 0.0000 \\
\hline & Second & 0.0000 & 1.0000 & 0.0004 & 0.0065 & 0.0026 & 0.0003 \\
\hline & Third & 0.0005 & 0.0008 & 0.9999 & 0.2025 & 0.0385 & 0.0000 \\
\hline & Fourth & 0.0000 & 0.0065 & 0.1975 & 1.0000 & 0.5928 & 0.0004 \\
\hline & Fifth & 0.0000 & 0.0021 & 0.0457 & 0.5826 & 0.9999 & 0.0001 \\
\hline & Sixth & 0.0000 & 0.0003 & 0.0000 & 0.0004 & 0.0001 & 1.0000 \\
\hline
\end{tabular}

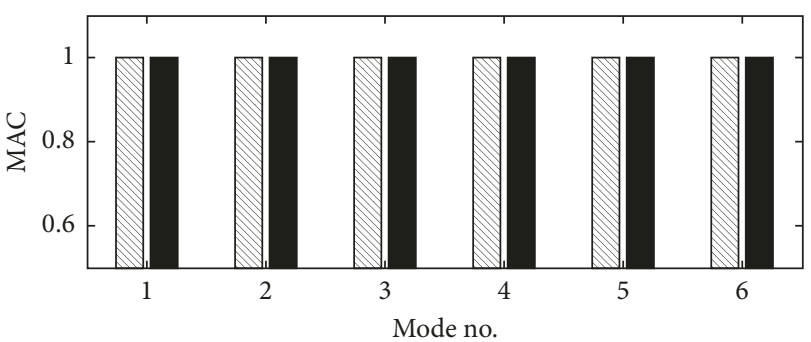

MSCP method with damage

Considering errors with damage

Figure 10: Comparison results of the MAC values in the Wangyu River Bridge (D-I).

\section{Conclusions}

This paper proposes the mass-stiffness combined perturbation method for monitoring the mode shapes of damaged structures. A numerical analysis and a laboratory test are conducted to demonstrate the effectiveness of the MSCP method. In addition, a concrete continuous bridge is analyzed to illustrate the application of the proposed technique to real structures and to encourage its implementation into bridge health monitoring systems.

The analysis of these numerical, experimental, and practical cases showed that the proposed MSCP method works well for structures with small damage severity. Therefore, this method is especially suitable for identifying mode shapes under minor damage scenarios.

Compared with the existing methods in the literature, the proposed technique maintains the following main advantages: (1) the errors in the identification of mode shapes are avoided as there is no need to monitor and identify mode shapes of damaged structures and (2) the monitoring procedure turns more practically convenient and accurate since only the vibration frequency needs to be measured, and meanwhile, it keeps the high precision on identification of the mode shapes.

\section{Notations}

$K^{u}$ : Global stiffness matrix for the intact structure

$M^{u}$ : Global mass matrix for the intact structure

$\lambda_{i}^{u}$ : The $i^{\text {th }}$ eigenvalue (or frequency) for the intact structure

$\phi_{i}^{u}$ : The $i^{\text {th }}$ mode shape for the intact structure

$K^{d}$ : Global stiffness matrix for the damaged structure

$M^{d}$ : Global mass matrix for the damaged structure

$\lambda_{i}^{d}$ : The $i^{\text {th }}$ eigenvalue (or frequency) for the damaged structure

$\phi_{i}^{d}$ : The $i^{\text {th }}$ mode shape for the damaged structure

$\Delta K$ : Change in the global stiffness matrix

$\Delta M: \quad$ Change in the global mass matrix

$\Delta \lambda_{i}: \quad$ Change in the $i^{\text {th }}$ eigenvalue (or frequency)

$\Delta \phi_{i}$ : Change in the $i^{\text {th }}$ mode shapes

$\Delta \lambda_{i, K}$ : The change in the $i^{\text {th }}$ eigenvalue (or frequency) due to the change in stiffness

$\Delta \phi_{i, K}$ : The change in the $i^{\text {th }}$ mode shape due to the change in stiffness

$\Delta \lambda_{i, M}$ : The change in the $i^{\text {th }}$ eigenvalue (or frequency) due to the change in mass

$\Delta \phi_{i, M}$ : The change in the $i^{\text {th }}$ mode shape due to the change in mass

$\lambda_{i, K}: \quad$ The $i^{\text {th }}$ frequency due to stiffness perturbation

$\lambda_{i, M}$ : The $i^{\text {th }}$ frequency due to mass perturbation

$F_{\text {obj }}$ : An objective function for selecting mass perturbation model. 


\section{Data Availability}

Most of the data used to support the findings of this study are available in the manuscripts, and more detailed data could be obtained from the corresponding author upon request.

\section{Conflicts of Interest}

The authors declare that they have no conflicts of interest.

\section{Acknowledgments}

This work was supported by the National Key R\&D Program of China (2018YFC0809606), National Natural Science Foundation of China (51978508), Beijing Natural Science Foundation (8192046), and Project of Basic Scientific Research Operating Expenses of Research Institute of Highway Ministry of Transport (2019-0102).

\section{References}

[1] D. Wang, W. Xiang, and H. Zhu, "Damage identification in beam type structures based on statistical moment using a two step method," Journal of Sound and Vibration, vol. 333, no. 3 , pp. 745-760, 2014.

[2] Z. Ismail, Z. Ibrahim, A. Z. C. Ong, and A. G. A. Rahman, "Approach to reduce the limitations of modal identification in damage detection using limited field data for nondestructive structural health monitoring of a cable-stayed concrete bridge," Journal of Bridge Engineering, vol. 17, no. 6, pp. 867-875, 2012.

[3] Y. Xia, H. Nassif, E.-S. Hwang, and D. Linzell, "Optimization of design details in orthotropic steel decks subjected to static and fatigue loads," Transportation Research Record: Journal of the Transportation Research Board, vol. 2331, no. 1, pp. 14-23, 2013.

[4] Y. J. Yan, H. F. Yang, Z. Y. Wu, and X. Ge, "Damage detection method for composite structures based on a combined technique of cross modal strain energy and Niche genetic algorithms," Journal of Vibration and Control, vol. 16, no. 11, pp. 1673-1683, 2010.

[5] H. R. Ahmadi, F. Daneshjoo, and N. Khaji, "New damage indices and algorithm based on square time-frequency distribution for damage detection in concrete piers of railroad bridges," Structural Control and Health Monitoring, vol. 22, no. 1, pp. 91-106, 2015.

[6] Y. G. Zhang, C. W. Liu, and J. Z. Wu, "The application of the three-step location approach in damage identification of the spatial lattice structure," Disaster Advances, vol. 6, pp. 74-80, 2013.

[7] V. B. Dawari and G. R. Vesmawala, "Structural damage identification of beam structures using two stage method based on modal strain energy indicators and artificial neural networks," Journal of Vibroengineering, vol. 18, pp. 119-128, 2016.

[8] O. S. Salawu, "Detection of structural damage through changes in frequency: a review," Engineering Structures, vol. 19, no. 9, pp. 718-723, 1997.

[9] R. Bolton, C. Sikorsky, S. Park, S. Choi, and N. Stubbs, "Modal property changes of a seismically damaged concrete bridge," Journal of Bridge Engineering, vol. 10, no. 4, pp. 415-428, 2005.

[10] P. Simon, A. Goldack, and S. Narasimhan, "Mode shape expansion for lively pedestrian bridges through kriging,"
Journal of Bridge Engineering, vol. 21, no. 6, Article ID 04016015, 2016.

[11] F. Vestroni and D. Capecchi, "Damage detection in beam structures based on frequency measurements," Journal of Engineering Mechanics, vol. 126, no. 7, pp. 761-768, 2000.

[12] J.-T. Kim, Y.-S. Ryu, H.-M. Cho, and N. Stubbs, "Damage identification in beam-type structures: frequency-based method vs mode-shape-based method," Engineering Structures, vol. 25, no. 1, pp. 57-67, 2003.

[13] A. Dutta and S. Talukdar, "Damage detection in bridges using accurate modal parameters," Finite Elements in Analysis and Design, vol. 40, no. 3, pp. 287-304, 2004.

[14] I. Mekjavić, "Identification of structural damage in bridges using high-frequency vibrational responses," Shock and Vibration, vol. 2015, Article ID 906062, 8 pages, 2015.

[15] X. T. Zhou, Y. Q. Ni, and F. L. Zhang, "Damage localization of cable-supported bridges using modal frequency data and probabilistic neural network," Mathematical Problems in Engineering, vol. 2014, Article ID 837963, 10 pages, 2014.

[16] J. Li, H. Hao, Y. Xia, and H. P. Zhu, "Damage detection of shear connectors in bridge structures with transmissibility in frequency domain," International Journal of Structural Stability and Dynamics, vol. 14, no. 2, Article ID 1350061, 2014.

[17] P. Cawley and R. D. Adams, "The location of defects in structures from measurements of natural frequencies," The Journal of Strain Analysis for Engineering Design, vol. 14, no. 2, pp. 49-57, 1979.

[18] X. Y. Zhao, Z.-Q. Lang, G. Park et al., “A new transmissibility analysis method for detection and location of damage via nonlinear features in MDOF structural systems," IEEE/ASME Transactions on Mechatronics, vol. 20, no. 4, pp. 1933-1947, 2015.

[19] W. Yang, Z. Lang, and W. Tian, "Condition monitoring and damage location of wind turbine blades by frequency response transmissibility analysis," IEEE Transactions on Industrial Electronics, vol. 62, no. 10, pp. 6558-6564, 2015.

[20] J.-W. Xiang, T. Matsumoto, J.-Q. Long, and G. Ma, "Identification of damage locations based on operating deflection shape," Nondestructive Testing and Evaluation, vol. 28, no. 2, pp. 166-180, 2013.

[21] Q. Lu, G. Ren, and Y. Zhao, "Multiple damage location with flexibility curvature and relative frequency change for beam structures," Journal of Sound and Vibration, vol. 253, no. 5, pp. 1101-1114, 2002.

[22] T. Contursi, A. Messina, and E. J. Williams, "A multipledamage location assurance criterion based on natural frequency changes," Journal of Vibration and Control, vol. 4, no. 5, pp. 619-633, 1998.

[23] C. C. Chang, T. Y. P. Chang, and Q. W. Zhang, "Ambient vibration of long-span cable-stayed bridge," Journal of Bridge Engineering, vol. 6, no. 1, pp. 46-53, 2001.

[24] L. Zhang and L. Sun, "Effect of concrete carbonation on natural frequency of reinforced concrete beams," Advances in Structural Engineering, vol. 20, no. 3, pp. 316-330, 2016.

[25] H. A. Razak and F. C. Choi, "The effect of corrosion on the natural frequency and modal damping of reinforced concrete beams," Engineering Structures, vol. 23, no. 9, pp. 1126-1133, 2001.

[26] T. Liu, Q. Zhang, T. Zordan, and B. Briseghella, "Finite element model updating of Canonica bridge using experimental modal data and genetic algorithm," Structural Engineering International, vol. 26, no. 1, pp. 27-36, 2016.

[27] R. M. Zhong, Z. H. Zong, Q. Q. Liu, and H. F. Zhou, “A multiscale finite element model validation method of 
composite cable-stayed bridge based on structural health monitoring system," Shock and Vibration, vol. 2015, Article ID 817281, 12 pages, 2015.

[28] P. Moradipour, T. H. T. Chan, and C. Gallag, "An improved modal strain energy method for structural damage detection, 2D simulation," Structural Engineering and Mechanics, vol. 54, no. 1, pp. 105-119, 2015.

[29] B. Wei, C. Zuo, X. He, L. Jiang, and T. Wang, "Effects of vertical ground motions on seismic vulnerabilities of a continuous track-bridge system of high-speed railway," Soil Dynamics and Earthquake Engineering, vol. 115, pp. 281-290, 2018.

[30] B. Wei, C. Zuo, X. He, and L. Jiang, "Numerical investigation on scaling a pure friction isolation system for civil structures in shaking table model tests," International Journal of Nonlinear Mechanics, vol. 98, pp. 1-12, 2018.

[31] M. Sanayei, B. Arya, E. M. Santini, and S. Wadia-Fascetti, "Significance of modeling error in structural parameter estimation," Computer-Aided Civil And Infrastructure Engineering, vol. 16, no. 1, pp. 12-27, 2001.

[32] A. Esfandiari, F. Bakhtiari-Nejad, and A. Rahai, "Theoretical and experimental structural damage diagnosis method using natural frequencies through an improved sensitivity equation," International Journal of Mechanical Sciences, vol. 70, pp. 79-89, 2013.

[33] M. Nacim, O. Mohammed, D. Mourad, and M. Brahim, "Experimental damage localization in beam by using natural frequency distribution and modal strain energy change ratio based methods," Journal of Vibroengineering, vol. 17, no. 3, pp. 1203-1217, 2015.

[34] O. Huth, G. Feltrin, J. Maeck, N. Kilic, and M. Motavalli, "Damage identification using modal data: experiences on a prestressed concrete bridge," Journal of Structural Engineering, vol. 131, no. 12, pp. 1898-1910, 2005.

[35] J. Derezinski, V. Jaksic, and C. A. Pillet, "Perturbation theory of W*-dynamics, liouvilleans and KMS-states," Reviews in Mathematical Physics, vol. 15, no. 5, pp. 447-489, 2003.

[36] Y. M. Zhang, S. H. Chen, Q. L. Liu, and T. Q. Liu, "Stochastic perturbation finite elements," Computers \& Structures, vol. 59, pp. 425-429, 1996.

[37] G. Ghosh and S. Ray-Chaudhuri, "Location sensitivity of fundamental and higher mode shapes in localization of damage within a building," Journal of Sound and Vibration, vol. 365, pp. 244-259, 2016.

[38] G. R. Gillich, I. C. Mituletu, I. Negru, M. Tufoi, V. Iancu, and F. Muntean, "A method to enhance frequency readability for early damage detection," Journal of Vibration Engineering \& Technologies, vol. 3, pp. 637-652, 2015.

[39] L. Thien-Phu and P. Argoul, "Modal identification using the frequency-scale domain decomposition technique of ambient vibration responses," Journal of Sound and Vibration, vol. 384, pp. 325-338, 2016.

[40] C. S. Huang, "Structural identification from ambient vibration measurement using the multivariate AR model," Journal of Sound and Vibration, vol. 241, no. 3, pp. 337-359, 2001.

[41] P. Duhamel and M. Vetterli, "Fast fourier transforms: a tutorial review and a state of the art," Signal Processing, vol. 19, no. 4, pp. 259-299, 1990.

[42] S. Lei, K. Mao, L. Li, W. Xiao, and B. Li, "Sensitivity analysis of modal assurance criteria of damped systems," Journal of Vibration and Control, vol. 23, no. 4, pp. 632-644, 2017. 


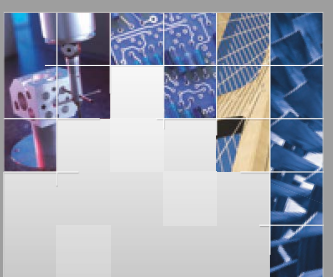

\section{Enfincering}
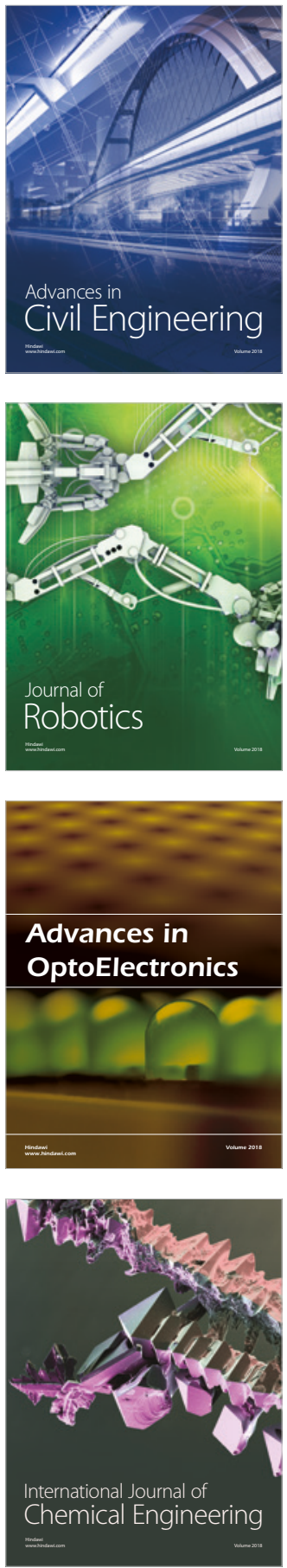

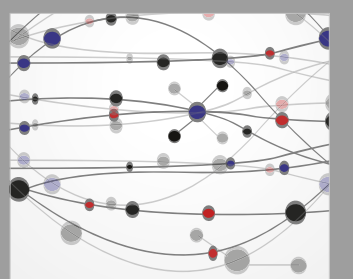

\section{Rotating \\ Machinery}

The Scientific World Journal

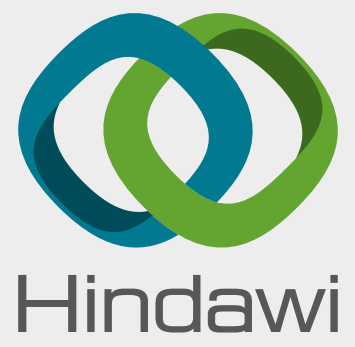

Submit your manuscripts at

www.hindawi.com
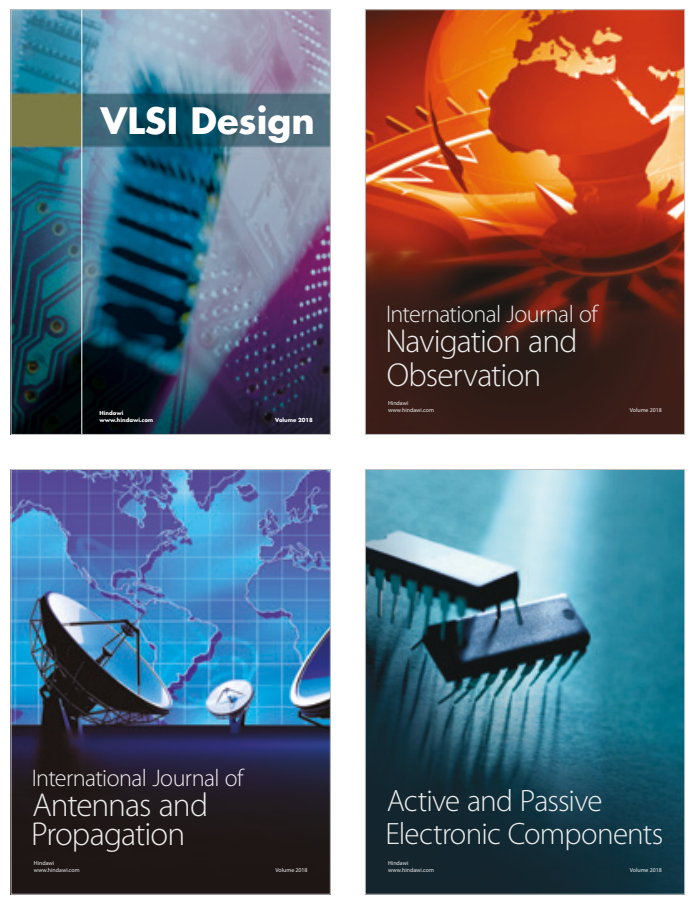
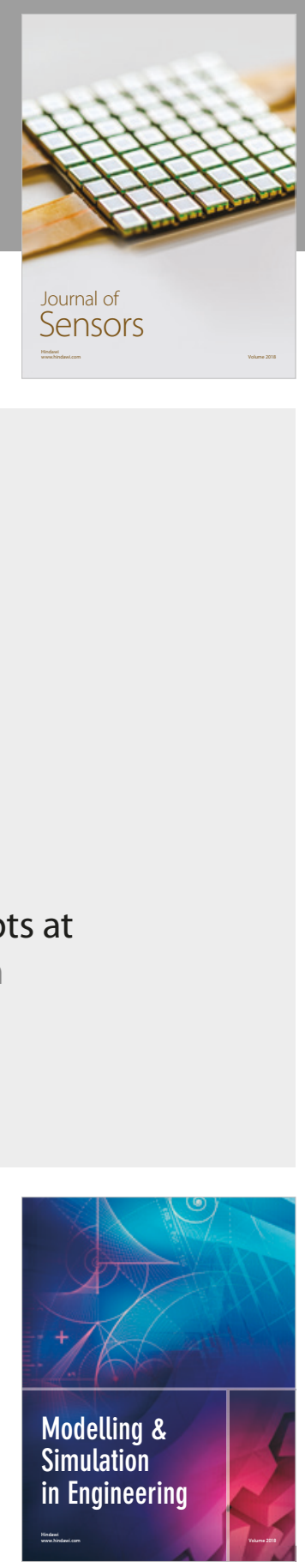

\section{Advances \\ Multimedia}
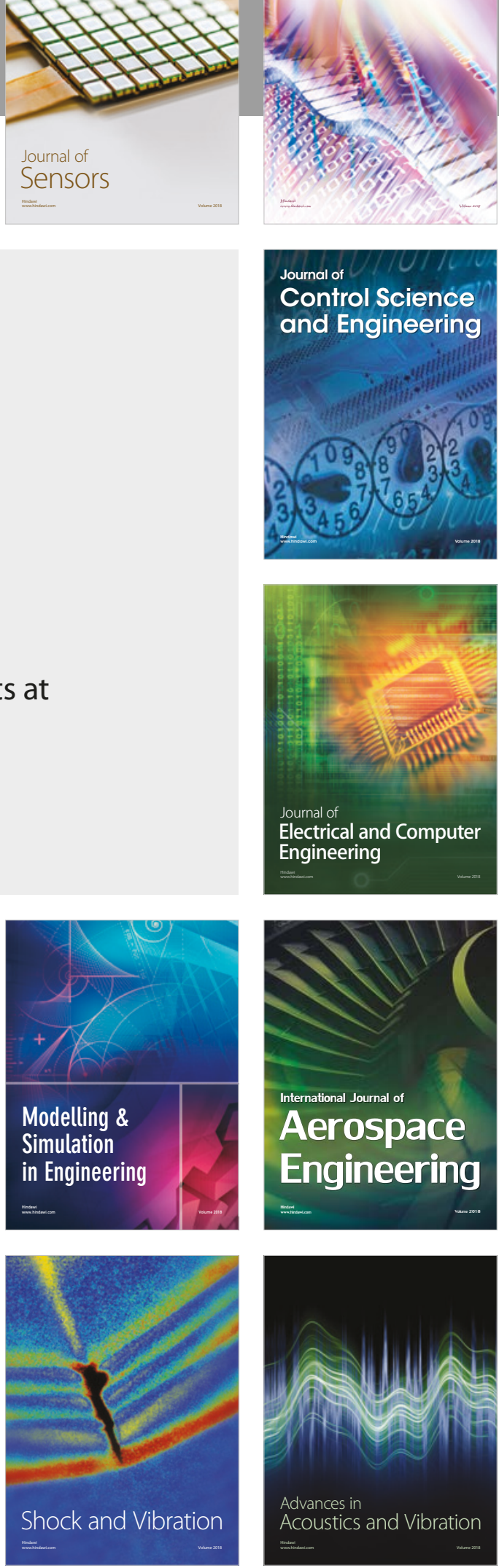